\title{
Traditional Korean Herbal Formula Samsoeum Attenuates Adipogenesis by Regulating the Phosphorylation of ERK1/2 in 3T3-L1 Cells
}

\author{
Soo-Jin Jeong, ${ }^{1,2}$ Sae-Rom Yoo, ${ }^{3}$ Chang-Seob Seo, ${ }^{3}$ and Hyeun-Kyoo Shin ${ }^{3}$ \\ ${ }^{1}$ KM Convergence Research Division, Korea Institute of Oriental Medicine, Daejeon 305-811, Republic of Korea \\ ${ }^{2}$ Korean Medicine Life Science, University of Science \& Technology, Daejeon 305-350, Republic of Korea \\ ${ }^{3}$ K-Herb Research Center, Korea Institute of Oriental Medicine, Daejeon 305-811, Republic of Korea
}

Correspondence should be addressed to Hyeun-Kyoo Shin; hkshin@kiom.re.kr

Received 11 May 2015; Revised 13 July 2015; Accepted 10 August 2015

Academic Editor: I-Min Liu

Copyright (C) 2015 Soo-Jin Jeong et al. This is an open access article distributed under the Creative Commons Attribution License, which permits unrestricted use, distribution, and reproduction in any medium, provided the original work is properly cited.

\begin{abstract}
Adipogenesis is the cell differentiation process from preadipocytes into adipocytes and the critical action in the development of obesity. In the present study, we conducted in vitro analyses to investigate the inhibitory effects of Samsoeum (SSE), a traditional herbal decoction. SSE had no significant cytotoxic effect against either the undifferentiated or differentiated 3T3-L1 cells. Oil Red O staining results showed that SSE significantly inhibited fat accumulation in adipocytes. SSE treatment consistently reduced the intracellular triglyceride content in the cells. SSE significantly inactivated glycerol-3-phosphate dehydrogenase (GPDH), a major link between carbohydrate and lipid metabolisms in 3T3-L1 adipocytes, and markedly inhibited the production of leptin, an important adipokine, in differentiated cells. SSE markedly suppressed the mRNA expression of the adipogenesis-related genes peroxisome proliferator-activated receptor-gamma $(P P A R-\gamma)$, CCAAT/enhancer binding protein-alpha $(C / E B P-\alpha)$, fatty acid synthase $(F A S)$, lipoprotein lipase ( $L P L)$, and fatty acid binding protein 4 (FABP4). Importantly, SSE increased the phosphorylation of ERK1/2, but not p38 MAPK and JNK, in adipose cells. Overall, our results indicate that SSE exerts antiadipogenic activity and modulates expressions of adipogenesis-related genes and ERK1/2 activation in adipocytes.
\end{abstract}

\section{Introduction}

Obesity is a major contributor to the development of type 2 diabetes, hyperglycemia, and cancer. Along with diet, exercise, behavior modification, and medications are used in the treatment of overweight or obese people. Among these treatments, medications are an important part of the treatment process for morbid obesity, but weight-loss drugs can have serious side effects. Sibutramine and orlistat have been used in the treatment of obesity for the past two decades. However, sibutramine, which suppresses the appetite, increases the risk of heart attack and stroke in patients with a history of cardiovascular disease $[1,2]$. Orlistat also has side effects including steatorrhea, dark urine, and stomach pain $[2,3]$. To overcome these limitations, a new approach to the treatment of obesity has been developed using complementary and alternative medicines such as herbal medicines.

Samsoeum (SSE, Shensuyin in Chinese, Jinsoin in Japanese), a traditional herbal medicine, was first recorded during the Song dynasty (China, AD 1107). SSE comprises 12 medicinal herbs including Perilla frutescens, Pueraria lobpt, Pinellia ternata, Angelica decursiva, Panax ginseng, Poria cocos, Citrus aurantium, Platycodon grandiflorum, Glycyrrhiza uralensis, Citrus unshiu, Zingiber officinale, and Zizyphus jujuba. It is used to treat the common cold, fever, and headache. Studies in recent years have established that SSE has pharmacological properties such as immune regulation, anticancer, and anti-inflammation [4-6]. SSE can also prevent allergic reactions after exposure to allergens and therefore may be an antiallergic agent $[7,8]$. Despite these 
observations, few scientific studies have examined its antiobesity effect.

In the present study, we evaluated inhibitory effect of SSE on 3T3-L1 adipocytes treated with SSE. We also investigated its mechanisms of action by examining its effects on the expressions of genes and proteins involved in lipid metabolisms.

\section{Materials and Methods}

2.1. Plant Materials. The 12 herbal medicines forming SSE were purchased from Omniherb (Yeongcheon, Korea) and HMAX (Jecheon, Korea). The origin of these herbal medicines was taxonomically confirmed by Professor Je Hyun Lee (Dongguk University, Gyeongju, Korea). A voucher specimen (2008-KE28-1 KE28-12) has been deposited at the K-herb Research Center, Korea Institute of Oriental Medicine.

2.2. Preparation of SSE Water Extract. SSE decoction comprising the 12 herbal medicines including Perillae Folium, Puerariae Radix, Pinelliae Tuber, Angelicae Decursive Radix, Ginseng Radix Alba, Poria Sclerotium, Aurantii Fructus Immaturus, Platycodonis Radix, Glycyrrhizae Radix et Rhizoma, Citri Unshius Pericarpium, Zingiberis Rhizoma Crudus, and Zizyphi Fructus was mixed (Table 1; $3.5 \mathrm{~kg}$; $41.25 \mathrm{~g} \times 85)$ and extracted in a 10 -fold mass of water at $100^{\circ} \mathrm{C}$ for $2 \mathrm{~h}$ under pressure $\left(1 \mathrm{kgf} / \mathrm{cm}^{2}\right)$ using an electric extractor (COSMOS-660; Kyungseo Machine Co., Incheon, Korea). The water extract was then filtered through a standard sieve (number 270, $53 \mu \mathrm{m}$; Chung Gye Sang Gong Sa, Seoul, Korea), and the solution was evaporated to dryness and freeze dried to give a powder. The yield of SSE water extract was $18.6 \%$ (651.4 g).

2.3. Cell Culture and Differentiation. The mouse 3T3-L1 preadipocyte cell line was obtained from the American Type Culture Collection (CL-173, ATCC, Rockville, MD). The cells were cultured in DMEM (Gibco BRL, Carlsbad, CA) supplemented with $10 \%$ newborn calf serum (Gibco BRL, Carlsbad, $\mathrm{CA})$ at $37^{\circ} \mathrm{C}$. For adipocyte differentiation, the cells were stimulated with 3T3-L1 differentiation medium containing isobutylmethylxanthine, dexamethasone, and insulin (MDI) (Zen-Bio Inc., Research Triangle Park, NC) for $48 \mathrm{~h}$ after reaching a confluent state. The medium was switched to DMEM containing $10 \% \mathrm{FBS}$ and $1 \mu \mathrm{g} / \mathrm{mL}$ insulin after 2 days and then changed to DMEM containing 10\% FBS for an additional 4 days. SSE extract was added to the cell culture during the 8 days of differentiation. GW9662 (Sigma-Aldrich, St. Louis, MO), PPAR- $\gamma$ antagonist, was used as positive control.

2.4. Cytotoxicity Assay. Undifferentiated 3T3-L1 cells were treated with various concentrations of SSE for $24 \mathrm{~h}$. To produce differentiated adipocyte cells, 3T3-L1 preadipocytes were differentiated for 8 days by stimulating them by SSE. CCK-8 solution (Dojindo, Kumamoto, Japan) was added, and the cells were incubated for $4 \mathrm{~h}$. After incubation, the absorbance was read at $450 \mathrm{~nm}$ on a microplate reader (Benchmark Plus, Bio-Rad. Hercules, CA).

2.5. Oil Red O Staining. The differentiated 3T3-L1 cells were fixed with $10 \%$ formalin for $15 \mathrm{~min}$ at room temperature and washed with $70 \%$ ethanol and PBS. The cells were stained with Oil Red O (Sigma-Aldrich, St. Louis, MO) for $5 \mathrm{~min}$ and then washed with PBS. Cell images were collected using an Olympus CKX41 inverted microscopy (Olympus, Tokyo, Japan). Stained oil droplets were dissolved in isopropyl alcohol and measured by reading the absorbance at $520 \mathrm{~nm}$ using microplate reader (Benchmark Plus, Bio-Rad. Hercules, CA).

2.6. Triglyceride Quantification Assay. The triglyceride concentration was measured enzymatically using a commercial kit (BioVision Inc., Milpitas, CA). Briefly, the 3T3-L1 adipocytes treated with SSE were homogenized in 5\% NP-40 assay buffer and the sample to solubilize all triglycerides. The sample was mixed with lipase and triglyceride reaction mixture. After a $1 \mathrm{~h}$ incubation, the sample absorbance was measured at $570 \mathrm{~nm}$ using microplate reader (Benchmark Plus, Bio-Rad. Hercules, CA).

2.7. Glycerol-3-Phosphate Dehydrogenase (GPDH) Activity Assay. After the induction of adipocyte differentiation with treating with SSE, 3T3-L1 cells were washed twice with PBS. GPDH activity was measured using a commercial kit (TAKARA, Tokyo, Japan) and by monitoring the dihydroxyacetone phosphate-dependent oxidation of $\mathrm{NADH}$ at $340 \mathrm{~nm}$. GPDH activity was expressed as unit/mg of protein.

2.8. Leptin Immunoassay. Leptin concentration was measured using a mouse leptin immunoassay kit (R\&D Systems, Minneapolis, MN) according to the manufacturer's instructions. In brief, the culture supernatant was collected from the differentiated 3T3-L1 adipocytes that has been treated with or without SSE. Equal amounts of the supernatants $(50 \mu \mathrm{L})$ and Assay Diluent RD1W (50 $\mu \mathrm{L})$ were added to a 96-well plate, and the plate was incubated for $2 \mathrm{~h}$ at room temperature. The plates were washed 5 times with $400 \mu \mathrm{L}$ of wash buffer; $100 \mu \mathrm{L}$ of mouse leptin conjugate was added to each well and incubated for $2 \mathrm{~h}$ at room temperature. The plates were washed 5 times; $100 \mu \mathrm{L}$ of substrate solution was added to each well and incubated for $30 \mathrm{~min}$ at room temperature in the dark. Finally, $100 \mu \mathrm{L}$ of stop solution was added to each well, and the absorbance was measured at $450 \mathrm{~nm}$ using microplate reader (Benchmark Plus, Bio-Rad. Hercules, CA).

2.9. RNA Isolation and Real-Time RT-PCR. Total RNA was prepared using TRIzol reagent (Invitrogen, Carlsbad, CA). Real-time RT-PCR analysis was performed using an Applied Biosystems 7300 Real-time PCR system and the SYBR green fluorescence quantification system (Applied Biosystems, Foster City, CA) to quantify the amplicons. cDNA was synthesized using $100 \mathrm{ng}$ of RNA in a reverse transcription reaction. The PCR conditions were 50 cycles of $95^{\circ} \mathrm{C}(30 \mathrm{~s}), 55^{\circ} \mathrm{C}(30 \mathrm{~s})$, and a standard denaturation curve. The primer sequences are listed in the $5^{\prime}$ to $3^{\prime}$ orientation in Table 2 . The PCR conditions 
TABle 1: Composition of Samsoeum (SSE).

\begin{tabular}{lccc}
\hline Herbal medicine & Scientific name & Supplier & Source \\
\hline Perillae Folium & Perilla frutescens & Omniherb & Geochang, Korea \\
Puerariae Radix & Pueraria lobata & Omniherb & Jecheon, Korea \\
Pinelliae Tuber & Pinellia ternata & HMAX & China \\
Angelicae Decursivae Radix & Angelica decursiva & HMAX & China \\
Ginseng Radix Alba & Panax ginseng & Omniherb & Geumsan, Korea \\
Poria Sclerotium & Poria cocos & Omniherb & Yeongcheon, Korea \\
Aurantii Fructus Immaturus & Citrus aurantium & HMAX & China \\
Platycodonis Radix & Platycodon grandiflorum & Omniherb & Yeongcheon, Korea \\
Glycyrrhizae Radix et Rhizoma & Glycyrrhiza uralensis & HMAX & China \\
Citri Unshius Pericarpium & Citrus unshiu & Omniherb & Jeju, Korea \\
Zingiberis Rhizoma Crudus & Zingiber officinale & Omniherb & Yeongcheon, Korea \\
Zizyphi Fructus & Zizyphus jujuba & Omniherb & Yeongcheon, Korea \\
\hline Total amount & & & 2.75 \\
\hline
\end{tabular}

TABLE 2: List of primer sequences for real-time RT-PCR.

\begin{tabular}{|c|c|}
\hline Gene & Primer sequences \\
\hline \multirow{2}{*}{ GAPDH } & Forward $5^{\prime}$-ACAATGAATACGGCTACAGCAACAG-3' \\
\hline & Reverse $5^{\prime}$-GGTGGTCCAGGGTTTCTTACTCC-3' \\
\hline \multirow{2}{*}{$P P A R-\gamma$} & Forward $5^{\prime}$-TATGGAGTGACATAGAGTGTGCT-3' \\
\hline & Reverse $5^{\prime}$-CCACTTCAATCCACCCAGAAAG-3' \\
\hline \multirow{2}{*}{$C / E B P-\alpha$} & Forward $5^{\prime}$-CAAGAACAGCAACGAGTACCG-3' \\
\hline & Reverse $5^{\prime}$-GTCACTGGTCAACTCCAGCAC-3' \\
\hline \multirow{2}{*}{ FABP4 } & Forward $5^{\prime}$-CAAGAACAGCAACGAGTACCG-3' \\
\hline & Reverse $5^{\prime}$-GTCACTGGTCAACTCCAGCAC-3' \\
\hline \multirow{2}{*}{$L P L$} & Forward 5'-CTGCTGGCGTAGCAGGAAGT-3' \\
\hline & Reverse $5^{\prime}$-CTGGAAAGTGCCTCCATTG-3' \\
\hline \multirow{2}{*}{ FAS } & Forward $5^{\prime}$-CAAGAACAGCAACGAGTACCG- $3^{\prime}$ \\
\hline & Reverse $5^{\prime}$-GTCACTGGTCAACTCCAGCAC-3' \\
\hline
\end{tabular}

for each target were optimized according to the primer concentration, the absence of primer dimer formation, and the efficiency of amplification of both the target genes and the housekeeping control gene. PCR reactions mixture comprised $1 \mu \mathrm{L}$ of $\mathrm{cDNA}$ and $9.5 \mu \mathrm{L}$ of PCR master mix, which contained 2x SYBR Green, 10 pmole each of the forward and reverse primer, and $4.5 \mu \mathrm{L}$ of DEPC-treated distilled water in a final volume of $15 \mu \mathrm{L}$. To normalize the cDNA content of the samples, we used the comparative threshold $\left(\mathrm{C}_{\mathrm{T}}\right)$ cycle method, which includes normalization of the number of target gene copies versus the endogenous reference gene, $\mathrm{GAPDH}$. The $\mathrm{C}_{\mathrm{T}}$ is defined as the fractional cycle number at which the fluorescence generated by cleavage of the probe passes a fixed threshold baseline when amplification of the PCR products is first detected.

2.10. Western Blotting. The protein was extracted using Mammalian Cell Lysis Buffer (Sigma-Aldrich, St. Louis, MO) containing protease inhibitor cocktail (Roche Applied Science, Indianapolis, IN). The protein concentration was measured using a protein assay reagent (Bio-Rad Lab, Hercules, CA). The proteins were resolved by $8-12 \%$ SDS-PAGE gels and transferred to polyvinylidene fluoride membranes (Millipore, Billerica, MA). Nonspecific biding sites were blocked with $5 \%(\mathrm{w} / \mathrm{v})$ skim milk, and the membranes were incubated with the primary antibodies anti-phospho-p38, phosphoJNK, phospho-ERK1/2 (Cell Signaling Tech., Danvers, MA), and $\beta$-actin (Santa Cruz Biotechnology, Santa Cruz, CA). The membranes were washed three times in TBST and then incubated with secondary antibodies for $1 \mathrm{~h}$ at room temperature. Protein expression was detected by using an ECL system (Thermo Scientific, Rockford, IL).

2.11. Chemicals and Reagents for HPLC Analysis. Puerarin, daidzin, liquiritin, naringin, glycyrrhizin (all purity $\geq 98.0 \%$ ), and hesperidin (purity $\geq 92.0 \%$ ) were purchased from Wako Pure Chemical Industries, Ltd. (Osaka, Japan). Neohesperidin (purity $\geq 99.0 \%$ ) was obtained from ChromaDex (Irvine, CA). The HPLC-grade reagents methanol, acetonitrile, and water were obtained from J. T. Baker (Phillipsburg, NJ). Glacial acetic acid was obtained from Merck KGaA (Darmstadt, Germany).

2.12. Preparation of Standard and Sample Solutions for HPLC Analysis. A standard stock solution of the 7 compounds puerarin, daidzin, liquiritin, naringin, hesperidin, neohesperidin, and glycyrrhizin was dissolved in methanol at concentration of $1.0 \mathrm{mg} / \mathrm{mL}$. For HPLC analysis, $200 \mathrm{mg}$ of lyophilized SSE extract was dissolved in $20 \mathrm{~mL}$ of distilled water, and the solution was filtered through a SmartPor GHP $0.2 \mu \mathrm{m}$ syringe filter (Woongki Science, Seoul, Korea) and then injected into the HPLC system.

2.13. HPLC Analysis of SSE. Analysis of the 7 compounds in SSE sample was performed using a Shimadzu LC-20A HPLC system (Shimadzu Co., Kyoto, Japan) comprising a solvent delivery unit, an online degasser, a column oven, an autosampler, and a PDA detector. The data processor was 


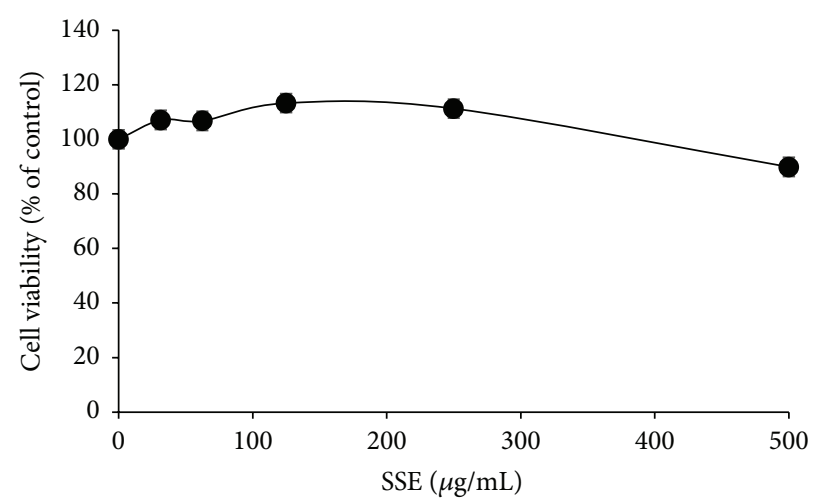

(a)

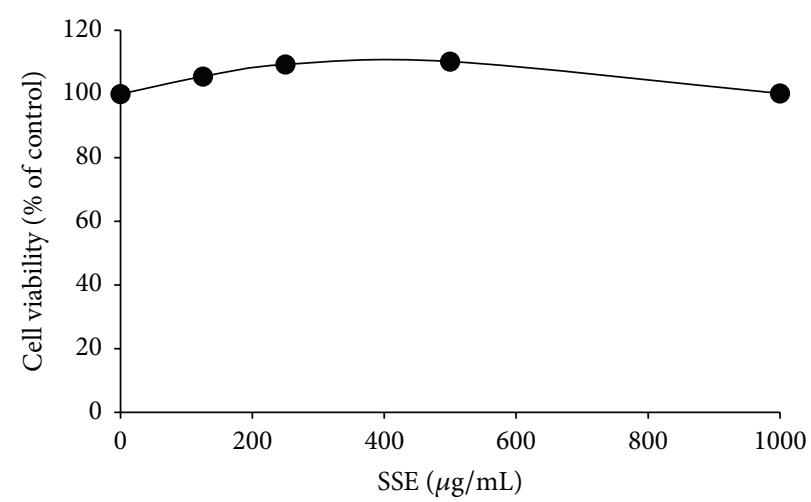

(b)

FIGURE 1: Cytotoxic effects of SSE extract in undifferentiated and differentiated 3T3-L1 cells. (a) 3T3-L1 preadipocytes were treated with various concentrations of SSE $(0,31.25,62.5,125,250$, or $500 \mu \mathrm{g} / \mathrm{mL})$ for $24 \mathrm{~h}$. (b) 3T3-L1 preadipocytes were differentiated into adipocytes by incubation with isobutylmethylxanthine, dexamethasone, and insulin (MDI) for 8 days. The cells were exposed to various concentrations of SSE $(0,62.5,125,250,500$, or $1000 \mu \mathrm{g} / \mathrm{mL})$ during the differentiation period. Cell viability was determined using a CCK-8 assay kit by measuring the absorbance at $450 \mathrm{~nm}$. Data are presented as mean $\pm \mathrm{SEM}$.

LCsolution software (version 1.24). The analytical column used was a Gemini $\mathrm{C}_{18}(250 \times 4.6 \mathrm{~mm}$; particle size $5 \mu \mathrm{m}$, Phenomenex, Torrance, CA) and maintained at $40^{\circ} \mathrm{C}$. The mobile phases comprised $1.0 \%(\mathrm{v} / \mathrm{v})$ acetic acid in water (A) and $1.0 \%(\mathrm{v} / \mathrm{v})$ acetic acid in acetonitrile (B). The gradient flow was as follows: $5-70 \% \mathrm{~B}$ for $0-40 \mathrm{~min}, 70-100 \% \mathrm{~B}$ for 40-45 $\mathrm{min}, 100 \%$ B for $45-50 \mathrm{~min}$, and $100-5 \%$ B for $55 \mathrm{~min}$. The flow rate was $1.0 \mathrm{~mL} / \mathrm{min}$, and the injection volume was $10 \mu \mathrm{L}$. The quantitative analysis of the 7 compounds was performed at $254 \mathrm{~nm}$ for puerarin, daidzin, and glycyrrhizin, and at $280 \mathrm{~nm}$ for liquiritin, naringin, hesperidin, and neohesperidin.

2.14. Statistical Analysis. All data were presented as mean \pm standard error of the mean (SEM). Group differences were assessed by one-way ANOVA and Tukey's multiple comparison post hoc test using GraphPad InStat ver.3.10 (GraphPad software Inc., San Diego, CA). The significance of the differences between the sample and normal control at $p<0.05$ or 0.01 was considered significant.

\section{Results}

3.1. Cytotoxic Effects of SSE against Undifferentiated and Differentiated 3T3-L1 Cells. To determine whether SSE had toxic effect, we used a CCK-8 assay to examine the cytotoxicity of SEE. Both preadipocytes and adipocytes were exposed to a concentration range of 31.5 to $1000 \mu \mathrm{g} / \mathrm{mL}$. As shown in Figure 1, SSE had no cytotoxic effect against 3T3-L1 preadipocytes (a) and adipocytes (b) compared with untreated cells. Nontoxic concentrations of the test materials were used for the subsequent experiments.

3.2. Effects of SSE on Adipogenesis in 3T3-L1 Cells. During adipogenesis, triglycerides are stored in the form of lipid droplets in adipocytes [9]. We used Oil Red O staining to examine the effects of SSE on lipid droplet accumulation. As shown in Figure 2, the number of lipid droplets increased markedly after differentiation for 8 days. Compared with the differentiated control cells, SSE-treated cells had significantly less intracellular lipid droplet accumulation (Figure 2(a)). To quantify the level of lipid accumulation, we dissolved the stained droplets in isopropyl alcohol and measured the optical density. Similar to the data in Figure 2(a), SSE inhibited lipid accumulation in a dose-dependent manner compared with the control group (Figure 2(b)). In parallel assay, intracellular triglyceride content was measured in SSEstimulated 3T3-L1 adipocytes. Consistent with the results of Oil Red O staining, triglyceride contents were increased significantly in the adipocytes, and SSE treatment decreased the accumulation of triglyceride compared with the differentiated cells (Figure 2(c)). At a maximum concentration of $400 \mu \mathrm{g} / \mathrm{mL}$, SSE significantly decreased lipid accumulation by up to $56 \%$ relative to the MDI-treated positive control group (Figure 2(c)). Similarly, GW9662 treatment used as a positive control markedly reduced triglyceride accumulation in adipocytes.

GPDH is an enzyme that generates glycerol-3-phosphate from dihydroxyacetone phosphate in adipocytes for lipid biosynthesis [10]. At a concentration of $25 \sim 400 \mu \mathrm{g} / \mathrm{mL}, \mathrm{SSE}$ treatment markedly reduced the GPDH activity compared with the cells differentiated into adipocytes (Figure 3(a)). The influence of SSE on the production of leptin, a major adipose hormone [11], was investigated in 3T3-L1 adipocytes. As shown in Figure 3(b), leptin production was inhibited by $>50 \%$ by SSE treatment at a concentration of 50 to $400 \mu \mathrm{g} / \mathrm{mL}$.

3.3. Effects of SSE on mRNA Expression of AdipogenesisRelated Genes in 3T3-L1 Adipocytes. Adipogenesis is accompanied by changes in the expression of adipogenesis-related transcriptional factors and specific molecular markers [12]. 


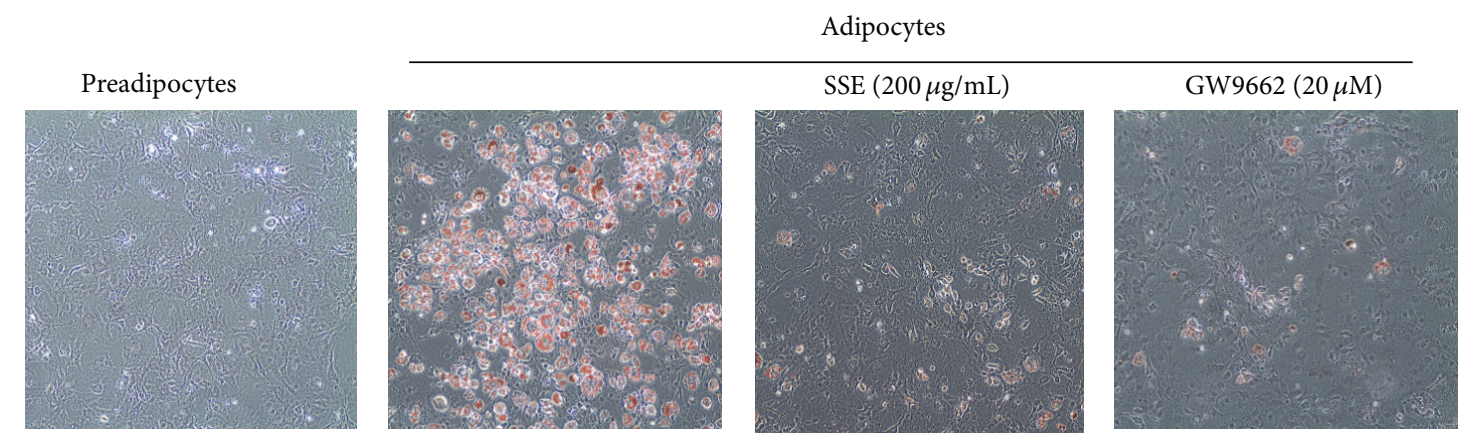

(a)

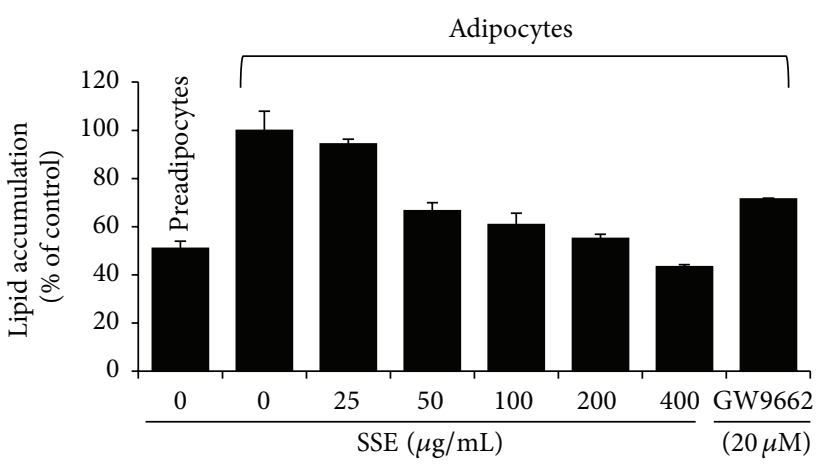

(b)

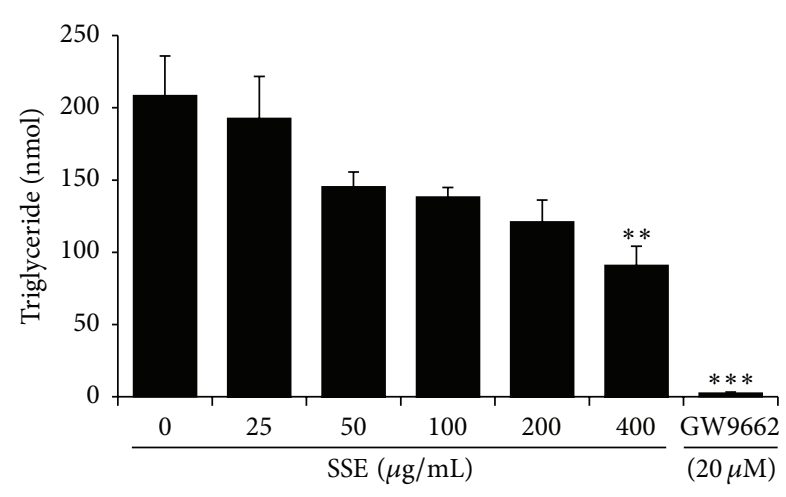

(c)

FIGURE 2: Inhibitory effect of SSE extract on triglyceride production in 3T3-L1 adipocytes. 3T3-L1 preadipocytes were differentiated into adipocytes by incubation with isobutylmethylxanthine, dexamethasone, and insulin (MDI) for 8 days. The cells were treated with or without SSE or GW9662 $(20 \mu \mathrm{M})$ during the differentiation period. ((a) and (b)) Lipid accumulation in the cells was analyzed by Oil Red O staining. (a) The stained cells were visualized on an Olympus CKX41 inverted microscopy at $\times 200$ of magnification. (b) Stained oil droplets were dissolved in isopropyl alcohol and quantified by reading the absorbance at $520 \mathrm{~nm}$. (c) The triglyceride content was measured enzymatically using a commercial kit (BioVision Inc.) at $570 \mathrm{~nm}$. Data are presented as mean \pm SEM. ${ }^{* *} p<0.01$ and ${ }^{* * *} p<0.001$ versus differentiated cells.

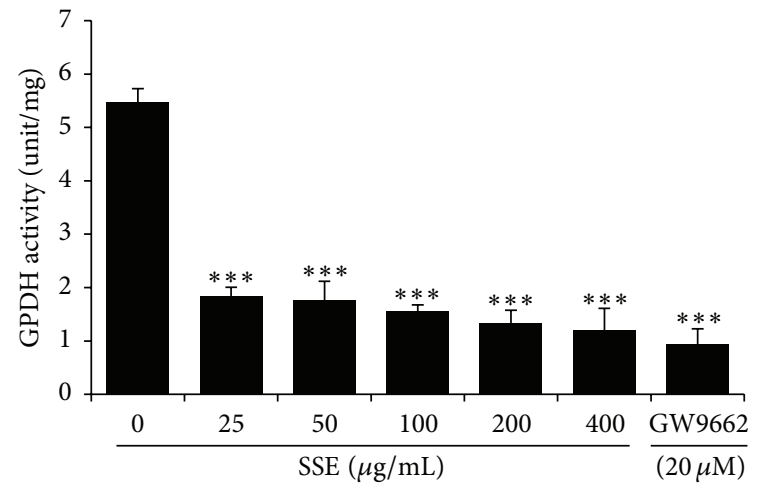

(a)

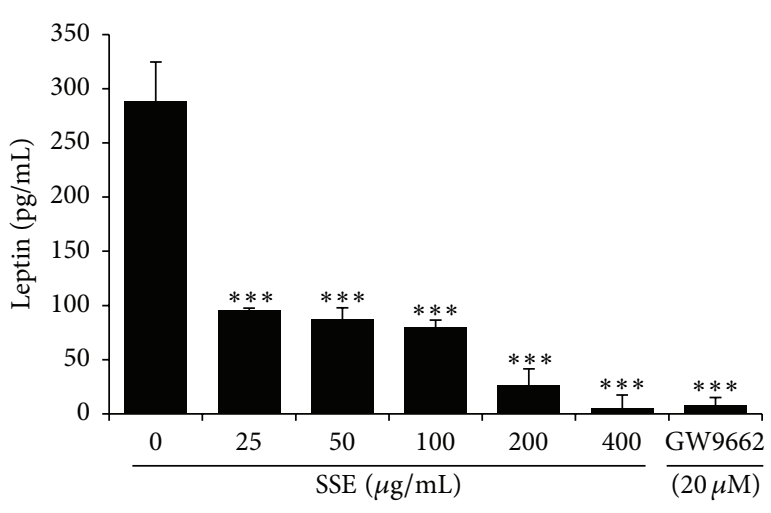

(b)

FIGURE 3: Inhibitory effects of SSE on GPDH activity and leptin production in 3T3-L1 adipocytes. 3T3-L1 preadipocytes were differentiated into adipocytes by incubation with isobutylmethylxanthine, dexamethasone, and insulin (MDI) for 8 days. The cells were exposed to various concentrations of SSE $(0,25,50,100,200$, or $400 \mu \mathrm{g} / \mathrm{mL})$ during the differentiation period. (a) GPDH activity of the cells was assessed by measuring the decrease in NADH at $340 \mathrm{~nm}$ using a TAKARA glycerol-3-phosphate dehydrogenase activity assay kit. (b) Culture supernatant was collected from the SSE-treated cells. Leptin production was determined by ELISA by subtracting the value measured at $450 \mathrm{~nm}$ using a mouse leptin immunoassay kit (R\&D Systems). Data are presented as the mean \pm SEM. ${ }^{* * *} p<0.01$ compared with the differentiated control. 


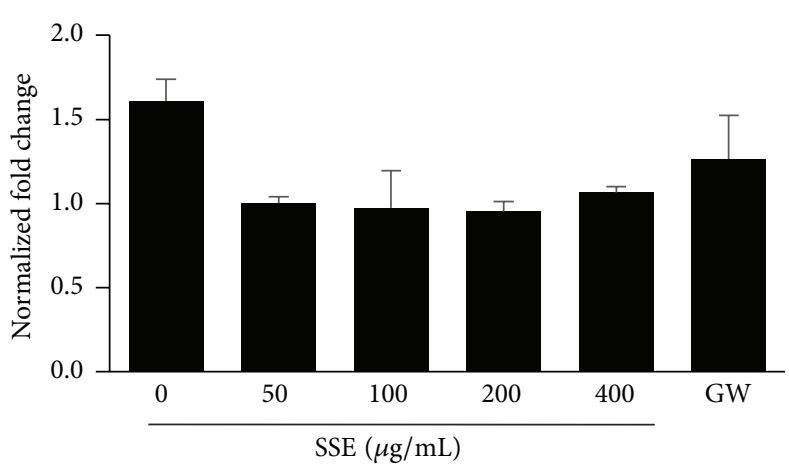

(a)

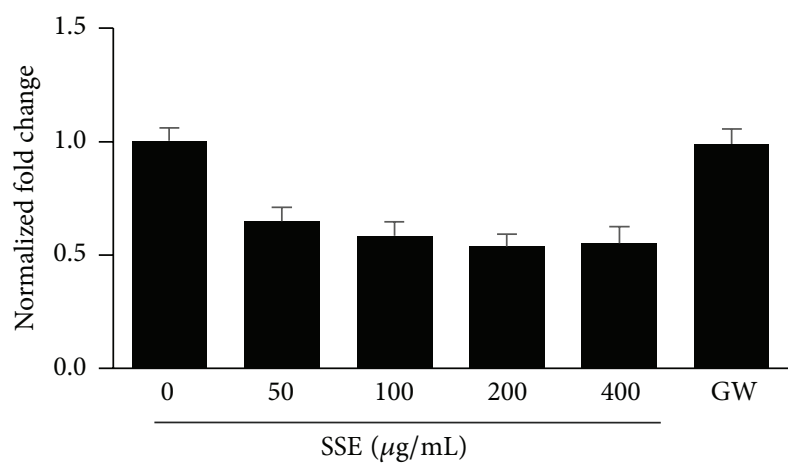

(c)

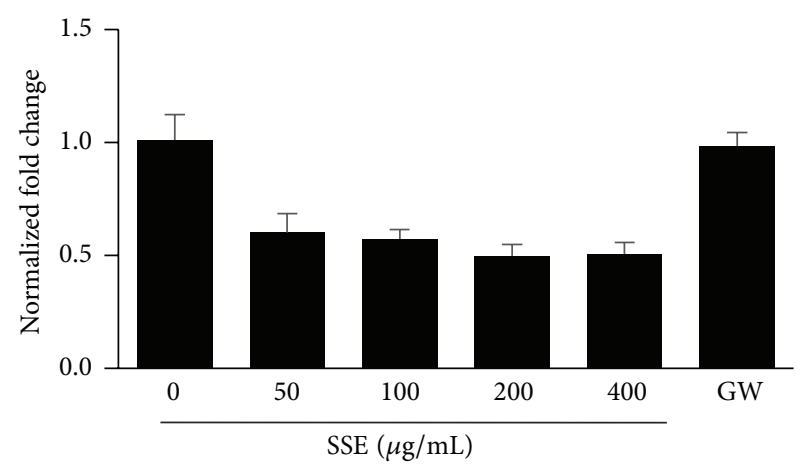

(b)

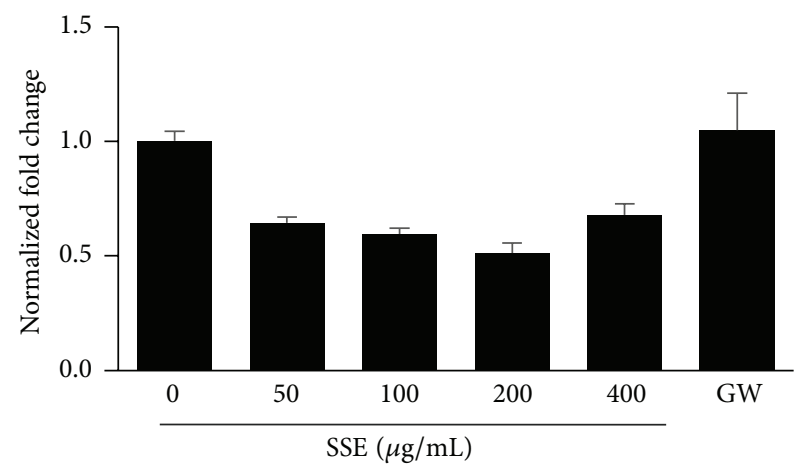

(d)

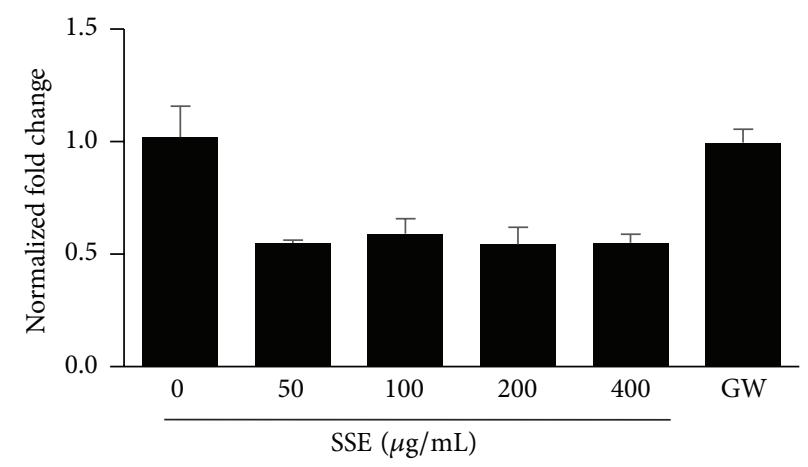

(e)

FIGURE 4: Effects of SSE on mRNA expression of lipid metabolism-related genes in 3T3-L1 adipocytes. (a) 3T3-L1 preadipocytes were differentiated into adipocytes by incubation with isobutylmethylxanthine, dexamethasone, and insulin (MDI) for 6 days. The cells were exposed to various concentrations of SSE $(0,25,50,100,200$, or $400 \mu \mathrm{g} / \mathrm{mL})$ during the differentiation period. Total RNA was isolated and subjected to real-time RT-PCR for PPAR- $\gamma$ (a) and C/EBP- $\alpha$ (b), FAS (c), LPL (d), and FABP4 (e). $\beta$-actin was used as a housekeeping gene.

PPAR- $\gamma$ and C/EBP- $\alpha$ are major transcription molecules in the adipogenesis pathway $[13,14]$. As shown in Figures 4(a) and 4(b), SSE treatment reduced the mRNA expression of $P P A R-\gamma$ and $C / E B P-\alpha$ in the differentiated adipocytes. In particular, the SSE caused greater suppression of PPAR- $\gamma$ expression compared with the PPAR- $\gamma$ inhibitor GW9662 [15]. SSE also decreased the expression of PPAR $-\gamma$ target genes $F A S$, LPL, and FABP4 in 3T3-L1 adipocytes (Figures 4(c), $4(\mathrm{~d})$, and $4(\mathrm{e}))$.

3.4. Effects of SSE on the Phosphorylation of MAPKs in 3T3-L1 Adipocytes. The MAPK pathways play a role in the regulation of each step in the process of adipogenesis [16]. To examine whether SSE treatment could influence the MAPK pathway, western blotting was conducted using anti-phospho-MAPKs including ERK1/2, p38 MAPK, and JNK. As shown in Figures 5(a) and 5(b), the level of phospho-ERK1/2 was increased by SSE in 3T3-L1 adipocytes. By contrast, SSE treatment had no significant effect on the phosphorylation of p38 MAPK or JNK in the cells. To confirm importance of the ERK pathway in SSE inhibition of adipogenesis, we utilized an ERK inhibitor PD98059 with SSE treatment. As shown in Figure 5(c), cotreatment of SSE and PD98059 blocked SSEinduced phosphorylation of ERK1 in 3T3-L1 adipocytes. 


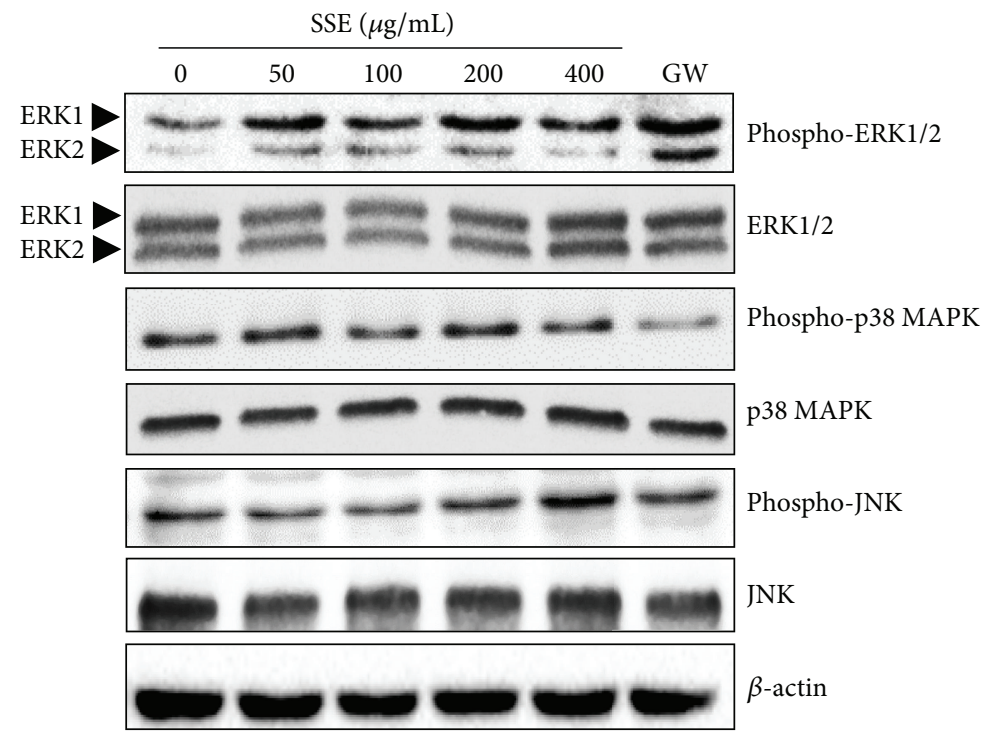

(a)
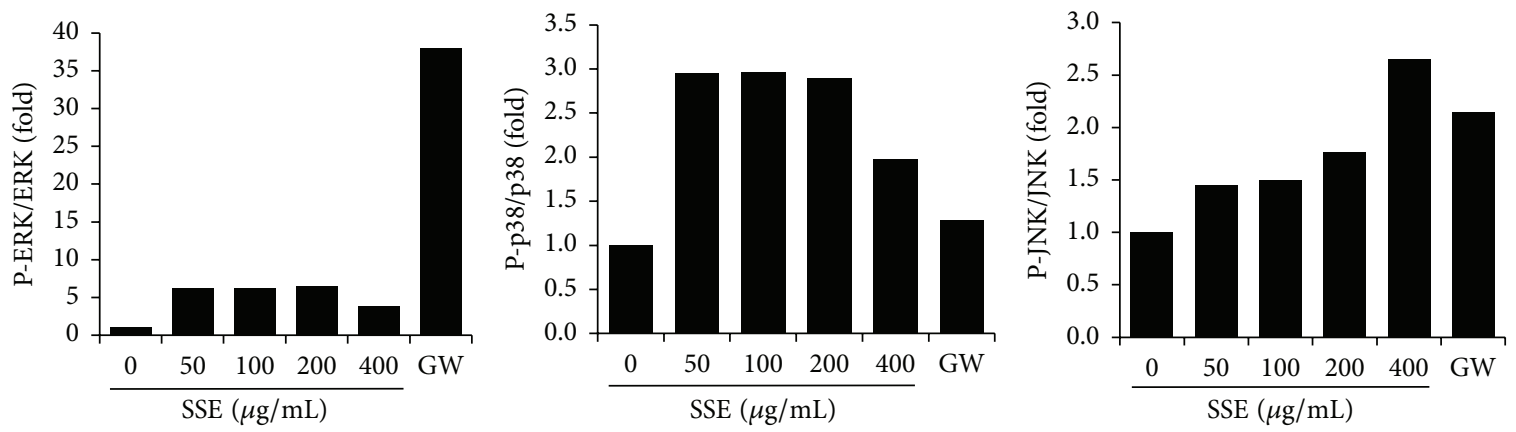

(b)

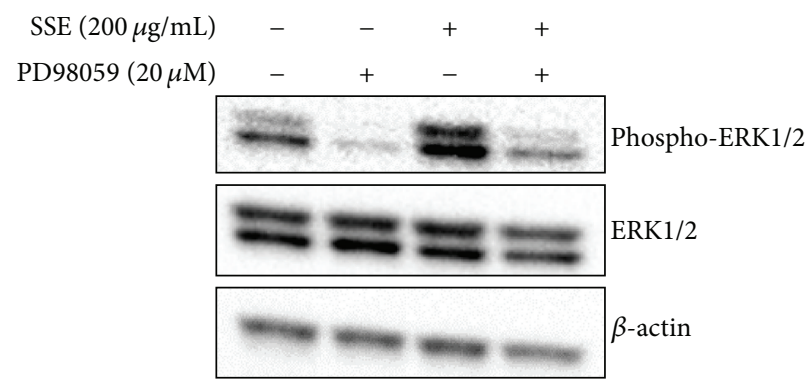

(c)

FIGURE 5: Effects of SSE on phosphorylation of the MAPK family proteins in 3T3-L1 adipocytes. (a) 3T3-L1 preadipocytes were differentiated into adipocytes by incubation with isobutylmethylxanthine, dexamethasone, and insulin (MDI) for 4 days. The cells were exposed to various concentrations of SSE $(0,25,50,100,200$, or $400 \mu \mathrm{g} / \mathrm{mL})$ during the differentiation period. Cell lysates were prepared and subjected to immunoblotting for phospho-ERK1/2, phospho-p38 MAPK, and phospho-JNK. (b) The graph represents the relative levels of phosphorylation of MAPKs. (c) 3T3-L1 cells were treated with SSE and/or ERK inhibitor PD98059. Cell lysates were prepared and subjected to immunoblotting for phospho-ERK1/2.

3.5. HPLC Analysis of SSE. We performed the simultaneous determination of seven components for quality control of SSE using HPLC coupled with photodiode array (PDA) detector. The regression equation of each compound was calculated by plotting the peak area $(y)$ against the concentrations $(x, \mu \mathrm{g} / \mathrm{mL})$ using mixed standard solutions. All calibration curves for quantitative analysis were obtained by assessment of the peak areas from standard solutions in the concentration ranges: puerarin, hesperidin, and neohesperidin, $1.56-200.00 \mu \mathrm{g} / \mathrm{mL}$; daidzin and liquiritin, $0.78-$ $100.00 \mu \mathrm{g} / \mathrm{mL}$; naringin, $1.95-250.00 \mu \mathrm{g} / \mathrm{mL}$; glycyrrhizin, $3.13-100.00 \mu \mathrm{g} / \mathrm{mL}$. The calibration curves for the $7 \mathrm{com}-$ pounds showed good linearity $\left(r^{2} \geq 0.9997\right)$. The limit of detection (LOD) and limit of quantitation (LOQ) of the 
TABLE 3: Regression data, linear range, correlation coefficient, LOD, and LOQ for marker compounds $(n=3)$.

\begin{tabular}{lccccc}
\hline Compound & Linear range $(\mu \mathrm{g} / \mathrm{mL})$ & Regression equation $^{\mathrm{a}}$ & ${\text { Correlation coefficient }\left(R^{2}\right)}^{\mathrm{LOD}^{\mathrm{b}}(\mu \mathrm{g} / \mathrm{mL})}$ & $\mathrm{LOQ}^{\mathrm{c}}\left(\mu \mathrm{g} / \mathrm{mL}^{2}\right.$ \\
\hline Puerarin & $1.56-200.00$ & $y=40369.09 x-4248.84$ & 0.9997 & 0.05 & 0.16 \\
Daidzin & $0.78-100.00$ & $y=37991.95 x+2862.21$ & 0.9998 & 0.05 & 0.06 \\
Liquiritin & $0.78-100.00$ & $y=18338.85 x-978.63$ & 1.0000 & 0.17 & 0.06 \\
Naringin & $1.95-250.00$ & $y=17333.29 x-2201.40$ & 0.9999 & 0.06 & 0.19 \\
Hesperidin & $1.56-200.00$ & $y=18669.49 x-2370.68$ & 0.0000 & 0.04 & 0.18 \\
Neohesperidin & $1.56-200.00$ & $y=23891.33 x-3109.41$ & 0.9999 & 0.14 \\
Glycyrrhizin & $3.13-100.00$ & $y=8064.35 x+1345.30$ & 1.74 \\
\hline
\end{tabular}

a $y$ : peak area (mAU) of compounds; $x$ : concentration $(\mu \mathrm{g} / \mathrm{mL})$ of compounds.

${ }^{\mathrm{b}} \mathrm{LOD}=3 \times$ signal-to-noise ratio.

${ }^{c}$ LOQ $=10 \times$ signal-to-noise ratio.

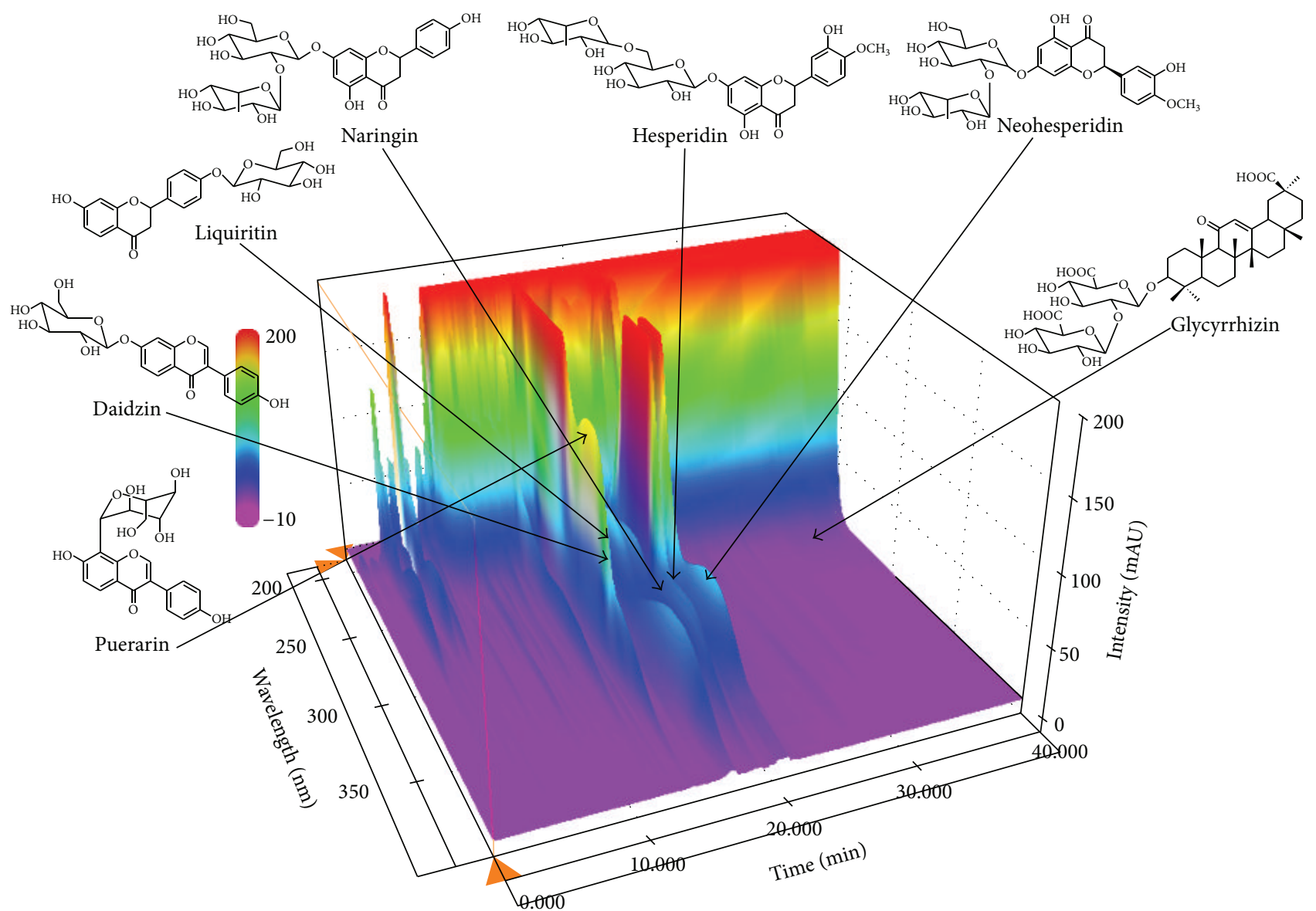

FIgURE 6: Three-dimensional chromatogram of SSE by HPLC-PDA. HPLC conditions, column: Gemini $\mathrm{C}_{18}$ column $(250 \times 4.6 \mathrm{~mm}, 5 \mu \mathrm{m}$; mobile phase: $1.0 \%(\mathrm{v} / \mathrm{v})$ acetic acid in water and $1.0 \%(\mathrm{v} / \mathrm{v})$ acetic acid in acetonitrile; gradient elution: $5-70 \%$ B for $0-40 \mathrm{~min}, 70-100 \%$ B for $40-45 \mathrm{~min}, 100 \%$ B for $45-50 \mathrm{~min}$, and $100-5 \%$ B for $55 \mathrm{~min}$; flow rate: $1.0 \mathrm{~mL} / \mathrm{min}$; column oven temperature: $40^{\circ} \mathrm{C}$; injection volume: $10 \mu \mathrm{L})$.

seven investigated compounds were $\leq 0.52$ and $1.74 \mu \mathrm{g} / \mathrm{mL}$, respectively (Table 3 ), which indicated that the analytical method was acceptable with satisfactory sensitivity. Using optimized chromatography conditions, three-dimensional chromatogram was obtained using an HPLC-PDA detector (Figure 6). The concentrations of 7 marker compounds were detected from 26 to $10.38 \mathrm{mg} / \mathrm{g}$, and these are summarized in Table 4.

\section{Discussion}

The field of herbal medicine includes the use and study of herbal plants for the purpose of preventing and treating various diseases. Herbs are attractive candidates for new drug development compared with synthetic chemical agents because they elicit fewer adverse effects of herbs. Recent studies have shown that many herbal plants have 
TABLE 4: Contents of seven compounds in the SSE by HPLC $(n=3)$.

\begin{tabular}{lcccc}
\hline Compound & Mean $(\mathrm{mg} / \mathrm{g})$ & SD & RSD $(\%)$ & Source $^{*}$ \\
\hline Puerarin & 5.29 & 0.01 & 0.22 & PR \\
Daidzin & 1.26 & 0.01 & 1.15 & PR \\
Liquiritin & 2.27 & 0.03 & 1.20 & GRR \\
Naringin & 10.38 & 0.05 & 0.49 & AFI, CUP \\
Hesperidin & 5.64 & 0.05 & 0.87 & AFI, CUP \\
Neohesperidin & 6.01 & 0.11 & 1.80 & AFI, CUP \\
Glycyrrhizin & 3.96 & 0.04 & 0.97 & GRR \\
\hline
\end{tabular}

* PR: Puerariae Radix, GRR: Glycyrrhizae Radix et Rhizoma, AFI: Aurantii Fructus Immaturus, and CUP: Citri Unshius Pericarpium.

antiobesity activity by regulating adipogenesis. Lee et al. reported antiobesity effects of Aster glehni extract in both in vitro and in vivo models [17]. Kwak et al. reported that Aristolochia manshuriensis Kom inhibited adipogenesis by regulating the ERK1/2 and Akt pathways [18]. Kubota et al. reported that Zizyphus jujuba extract blocked adipogenesis by targeting PPAR- $\gamma$ and C/EBP- $\alpha$ expression in 3T3-L1 cells [19]. Interestingly, the antiobesity effects of herbal formulas have also been reported in several recent papers [20-23]. An herbal formula is a mixture of several different herbs, which provides a greater efficiency, compared with each herb alone.

In the current study, we found that the traditional Korean herbal formula SSE inhibited adipogenesis in 3T3-L1 cells. Adipogenesis was induced by adding the differentiation stimulators MDI to 3T3-L1 preadipocytes. The differentiated adipose cells exhibited increased triglyceride accumulation, GPDH activation, and leptin production. By contrast, SSE treatment exerted strong inhibitory effects on triglyceride accumulation, GPDH activity, and leptin production in adipocytes (Figures 2 and 3 ).

Adipogenesis is a multistep process that is regulated by a cascade of various transcription factors. PPAR $\gamma$ and C/EBP$\alpha$ are key factors in the regulation of the expression of target genes leading to adipocyte development $[13,14,24]$. In our study, SSE markedly decreased the mRNA expression levels of $P P A R-\gamma$ and $C / E B P-\alpha$ in 3T3-L1 adipocytes. Consistently, SSE reduced the mRNA expression of the PPAR- $\gamma$ target genes $F A S, L P L$, and FABP4 (Figure 4). These data suggest that the principal mechanism responsible for the antiadipogenesis effects of SSE occurs through the inhibition of PPAR- $\gamma$ and $C / E B P-\alpha$ expression.

MAPK represents a family of proteins involved in various cellular processes such as cell survival, differentiation, and proliferation [25]. It is known that MAPKs regulate both normal and pathological adipogenesis [16]. ERK1/2 has both negative and positive effects on adipogenesis regulation, whereas p38 MAPK and JNK display limited effects. PPAR$\gamma, \mathrm{C} / \mathrm{EBP}-\alpha$, and the ERK pathway have been found to be involved in the regulation of each of adipogenesis [26]. The ERK pathway is necessary for the early proliferative steps of adipogenesis [27]. In addition, Hu et al. reported that the ERK pathway is known to have inhibitory effect on adipocyte maturation by targeting PPAR- $\gamma$ in the later steps of adipogenesis [28]. Consistently, we observed that the herbal formula SSE increased the phosphorylation of ERK in differentiation-induced 3T3-L1 cells. By contrast, other MAPK family members p38 MAPK and JNK were not significantly affected by SSE treatment (Figure 5). Furthermore, cotreatment of SSE and ERK inhibitor PD98059 confirmed importance of the ERK pathway antiadipogenesis of SSE. Together, our data suggest that inhibitory effect of SSE is dependent on the ERK pathway by targeting PPAR- $\gamma$ like $\mathrm{Hu}$ et al.s data [28]. Similar to our study, other studies of natural products including phytochemicals and herbal medicines have reported that their antiadipogenic potential involves targeting of the ERK1/2 pathway [18, 29, 30].

Antiobesity effects have been reported for 9 of the 12 SSE components except for Angelica decursiva, Poria cocos, and Glycyrrhiza uralensis [19, 31-37]. For example, Pueraria lobata improved impaired glucose and lipid metabolism in obese mice [32]. Pinellia ternata exhibited antiobesity effects through changes in thermogenesis and fatty acid oxidation [33]. Platycodon grandiflorum modified adipokines and the glucose uptake in high-fat diet- (HFD-) fed mice and in L6 muscle cells [35]. These findings strongly support the antiobesity activity of SSE and suggest its potential as an antiobesity drug candidate.

HPLC-PDA method is a convenient, widely used, and powerful approach for the rapid identification of constituents in botanical extracts and plants important in traditional Chinese medicine [38]. Hence, in this study, we performed quantitative determination of seven main components in SSE using HPLC-PDA. The investigated components were as follows: puerarin and daidzin form Puerariae Radix, liquiritin and glycyrrhizin from Glycyrrhizae Radix et Rhizoma, and naringin, hesperidin, and neohesperidin from Aurantii Fructus Immaturus and Citri Unshius Pericarpium. The optimized HPLC-PDA method was applied for simultaneous determination of the seven marker compounds in SSE. Among these components, naringin, which is a marker component of Aurantii Fructus Immaturus and Citri Unshius Pericarpium, was detected $10.38 \mathrm{mg} / \mathrm{g}$ as the main compounds compared with the others in SSE (Figure 6 and Table 3). The established HPLC-PDA method will be helpful to improve quality control of SSE.

In conclusion, our data demonstrate that SSE has the inhibitory effects on adipogenesis in 3T3-L1 adipocytes as indicated by a significant reduction in triglyceride accumulation without cytotoxicity. The inhibitory effects of SSE may be mediated through the suppression of PPAR- $\gamma$ and C/EBP$\alpha$ as well as activation of the ERK1/2 pathway. Further studies are needed to confirm the antiadipogenesis effects of SSE in obesity using HFD-fed obese animals. Herbal formulas have an advantage for multicompound/multitarget (MCMT) therapy because they are a cocktail drug comprising several different phytochemicals. The antiadipogenic effects of SSE could be expanded to drug development studies for possible treatment of obesity or other metabolic diseases.

\section{Conflict of Interests}

The authors have declared that no conflict of interests exists. 


\section{Acknowledgment}

This work was supported by a grant from the Korean Institute of Oriental Medicine (no. K14030).

\section{References}

[1] US Food and Drug Administration, Meridia (Sibutramine Hydrochloride): Follow-Up to an Early Communication about an Ongoing Safety Review, US Food and Drug Administration, 2010.

[2] E. M. van der Zwaal, S. K. Janhunen, M. C. M. Luijendijk et al., "Olanzapine and sibutramine have opposing effects on the motivation for palatable food," Behavioural Pharmacology, vol. 23, no. 2, pp. 198-204, 2012.

[3] H. Buyukhatipoglu, "A possibly overlooked side effect of orlistat: gastroesophageal reflux disease," Journal of the National Medical Association, vol. 100, no. 10, p. 1207, 2008.

[4] S. J. Kim, P. D. Moon, N. Y. Myung et al., "Samsoeum inhibits systemic anaphylaxis and release of histamine, cytokine in vivo and in vitro," Oriental Pharmacy and Experimental Medicine, vol. 9, no. 2, pp. 115-127, 2009.

[5] N. H. Ryu, S. M. Park, H. Kang et al., "Effect of samsoeum on cytokine regulation of mouse T cell," Korean Journal of Oriental Physiology \& Pathology, vol. 22, pp. 856-862, 2008.

[6] B.-S. Zhao, L.-F. Li, Y.-Y. Ma et al., "Effect of Shensu Yin on the expression of toll-like receptors and the downstream signaling components on RAW 264.7 cells," Zhongguo Zhong Yao Za Zhi, vol. 32, no. 4, pp. 327-332, 2007.

[7] S. J. Cho, H. W. Kim, B.-Y. Kim, and S. I. Cho, "Sam So Eum, a herb extract, as the remedy for allergen-induced asthma in mice," Pulmonary Pharmacology and Therapeutics, vol. 21, no. 3, pp. 578-583, 2008.

[8] B. S. Nam and Y. B. Ki, "The experimental study of Sam so eum and Sm so eum gamibang on anti-allergic effect," The Journal of Korean Oriental Medicine, vol. 15, pp. 343-345, 2002.

[9] W. Fei, X. Du, and H. Yang, "Seipin, adipogenesis and lipid droplets," Trends in Endocrinology and Metabolism, vol. 22, no. 6, pp. 204-210, 2011.

[10] L. P. Kozak and J. T. Jensen, "Genetic and developmental control of multiple forms of L-glycerol 3-phosphate dehydrogenase," The Journal of Biological Chemistry, vol. 249, no. 24, pp. 77757781, 1974.

[11] F. Zhang, M. B. Basinski, J. M. Beals et al., "Crystal structure of the obese protein leptin-E100," Nature, vol. 387, no. 6629, pp. 206-209, 1997.

[12] R. M. Cowherd, R. E. Lyle, and R. E. McGehee Jr., "Molecular regulation of adipocyte differentiation," Seminars in Cell \& Developmental Biology, vol. 10, no. 1, pp. 3-10, 1999.

[13] S. R. Farmer, "Regulation of PPARgamma activity during adipogenesis," International Journal of Obesity, vol. 29, supplement 1, pp. S13-S16, 2005.

[14] C. Semsarian, M.-J. Wu, Y.-K. Ju et al., "Skeletal muscle hypertrophy is mediated by a $\mathrm{Ca}^{2+}$-dependent calcineurin signalling pathway," Nature, vol. 400, no. 6744, pp. 576-581, 1999.

[15] L. M. Leesnitzer, D. J. Parks, R. K. Bledsoe et al., "Functional consequences of cysteine modification in the ligand binding sites of peroxisome proliferator activated receptors by GW9662," Biochemistry, vol. 41, no. 21, pp. 6640-6650, 2002.

[16] F. Bost, M. Aouadi, L. Caron, and B. Binétruy, "The role of MAPKs in adipocyte differentiation and obesity," Biochimie, vol. 87, no. 1, pp. 51-56, 2005.
[17] H.-M. Lee, G. Yang, T.-G. Ahn et al., "Antiadipogenic effects of Aster glehni extract: in vivo and in vitro effects", Evidence-Based Complementary and Alternative Medicine, vol. 2013, Article ID 859624, 10 pages, 2013.

[18] D. H. Kwak, J.-H. Lee, T. Kim et al., "Aristolochia manshuriensis Kom inhibits adipocyte differentiation by regulation of ERK1/2 and Akt pathway," PLoS ONE, vol. 7, no. 11, Article ID e49530, 2012.

[19] H. Kubota, R. Morii, A. Kojima-Yuasa, X. Huang, Y. Yano, and I. Matsui-Yuasa, "Effect of Zizyphus jujuba extract on the inhibition of adipogenesis in 3T3-L1 preadipocytes," American Journal of Chinese Medicine, vol. 37, no. 3, pp. 597-608, 2009.

[20] T. Liu, L.-L. Yang, L. Zou et al., "Chinese medicine formula Lingguizhugan decoction improves beta-oxidation and metabolism of fatty acid in high-fat-diet-induced rat model of fatty liver disease," Evidence-Based Complementary and Alternative Medicine, vol. 2013, Article ID 429738, 9 pages, 2013.

[21] S. S. Shin, D. Park, H. Y. Lee et al., "The herbal composition GGEx18 from Laminaria japonica, Rheum palmatum, and Ephedra sinica reduces obesity via skeletal muscle AMPK and PPAR $\alpha$, Pharmaceutical Biology, vol. 50, no. 4, pp. 506-515, 2012.

[22] S. S. Shin and M. Yoon, "The herbal composition GGEx18 from Laminaria japonica, Rheum palmatum, and Ephedra sinica inhibits high-fat diet-induced hepatic steatosis via hepatic PPAR $\alpha$ activation," Pharmaceutical Biology, vol. 50, no. 10, pp. 1261-1268, 2012.

[23] L. Yang, K. Lu, X.-Y. Wen et al., "Jueming Prescription reduces body weight by increasing the mRNA Expressions of beta3adrenergic receptor and uncoupling protein-2 in adipose tissue of diet-induced obese rats," Chinese Journal of Integrative Medicine, vol. 18, no. 10, pp. 775-781, 2012.

[24] S. R. Farmer, "Transcriptional control of adipocyte formation," Cell Metabolism, vol. 4, no. 4, pp. 263-273, 2006.

[25] G. Pearson, F. Robinson, T. B. Gibson et al., "Mitogen-activated protein (MAP) kinase pathways: regulation and physiological functions," Endocrine Reviews, vol. 22, no. 2, pp. 153-183, 2001.

[26] A. G. Cristancho and M. A. Lazar, "Forming functional fat: a growing understanding of adipocyte differentiation," Nature Reviews Molecular Cell Biology, vol. 12, no. 11, pp. 722-734, 2011.

[27] F. Bost, M. Aouadi, L. Caron et al., "The extracellular signalregulated kinase isoform ERK1 is specifically required for in vitro and in vivo adipogenesis," Diabetes, vol. 54, no. 2, pp. 402411, 2005.

[28] E. Hu, J. B. Kim, P. Sarraf, and B. M. Spiegelman, "Inhibition of adipogenesis through MAP kinase-mediated phosphorylation of PPAR $\gamma$," Science, vol. 274, no. 5295, pp. 2100-2103, 1996.

[29] J.-K. Kim, H. So, M.-J. Youn et al., "Hibiscus sabdariffa L. water extract inhibits the adipocyte differentiation through the PI3K and MAPK pathway," Journal of Ethnopharmacology, vol. 114, no. 2, pp. 260-267, 2007.

[30] T. Wang, Y. Wang, and H. Yamashita, "Evodiamine inhibits adipogenesis via the EGFR-PKC $\alpha$-ERK signaling pathway," FEBS Letters, vol. 583, no. 22, pp. 3655-3659, 2009.

[31] H.-J. Myoung, G. Kim, and K.-W. Nam, "Apigenin isolated from the seeds of Perilla frutescens britton var crispa (Benth.) inhibits food intake in C57BL/6J mice," Archives of Pharmacal Research, vol. 33, no. 11, pp. 1741-1746, 2010.

[32] J. K. Prasain, N. Peng, R. Rajbhandari, and J. M. Wyss, "The Chinese Pueraria root extract (Pueraria lobata) ameliorates impaired glucose and lipid metabolism in obese mice," Phytomedicine, vol. 20, no. 1, pp. 17-23, 2012. 
[33] Y.-J. Kim, Y.-O. Shin, Y.-W. Ha, S. Lee, J.-K. Oh, and Y. S. Kim, "Anti-obesity effect of Pinellia ternata extract in Zucker rats," Biological and Pharmaceutical Bulletin, vol. 29, no. 6, pp. 12781281, 2006.

[34] M. Y. Song, B. S. Kim, and H. Kim, "Influence of Panax ginseng on obesity and gut microbiota in obese middle-aged Korean women," Journal of Ginseng Research, vol. 38, no. 2, pp. 106-115, 2014.

[35] J. L. Verpeut, A. L. Walters, and N. T. Bello, “Citrus aurantium and Rhodiola rosea in combination reduce visceral white adipose tissue and increase hypothalamic norepinephrine in a rat model of diet-induced obesity," Nutrition Research, vol. 33, no. 6, pp. 503-512, 2013.

[36] S. Nammi, S. Sreemantula, and B. D. Roufogalis, "Protective effects of ethanolic extract of zingiber officinale rhizome on the development of metabolic syndrome in high-fat diet-fed rats," Basic and Clinical Pharmacology and Toxicology, vol. 104, no. 5, pp. 366-373, 2009.

[37] K. Takayanagi, S.-I. Morimoto, Y. Shirakura et al., "Mechanism of visceral fat reduction in Tsumura Suzuki obese, diabetes (TSOD) mice orally administered $\beta$-cryptoxanthin from Satsuma mandarin oranges (Citrus unshiu Marc)," Journal of Agricultural and Food Chemistry, vol. 59, no. 23, pp. 1234212351, 2011.

[38] H. Zhang, P. Shen, and Y. Cheng, "Identification and determination of the major constituents in traditional Chinese medicine Si-Wu-Tang by HPLC coupled with DAD and ESI-MS," Journal of Pharmaceutical and Biomedical Analysis, vol. 34, no. 3, pp. 705-713, 2004. 


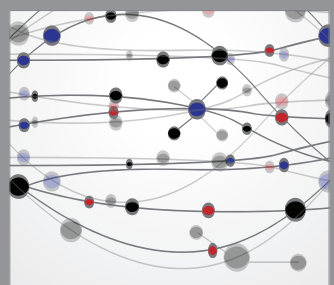

The Scientific World Journal
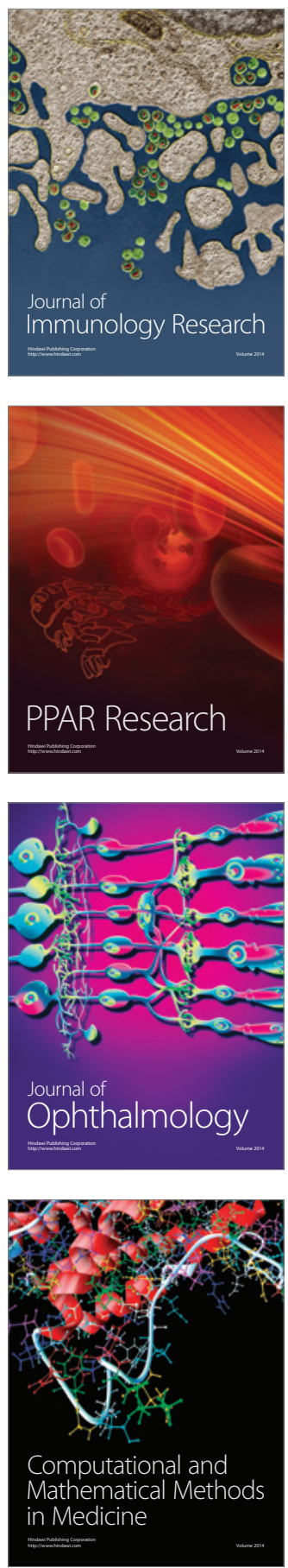

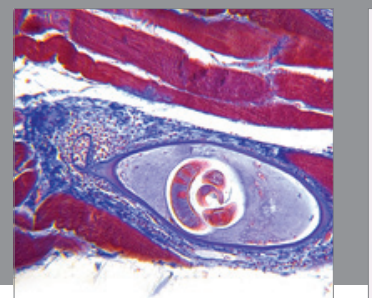

Gastroenterology

Research and Practice
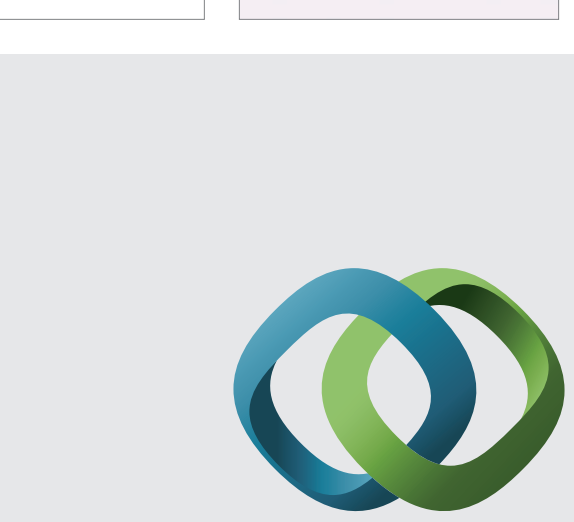

\section{Hindawi}

Submit your manuscripts at

http://www.hindawi.com
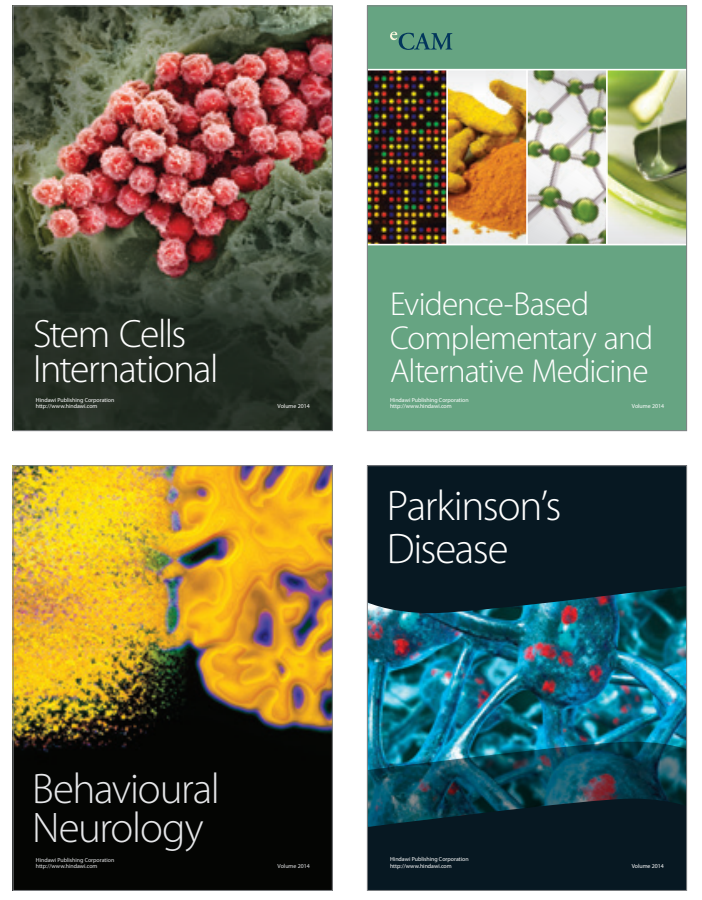
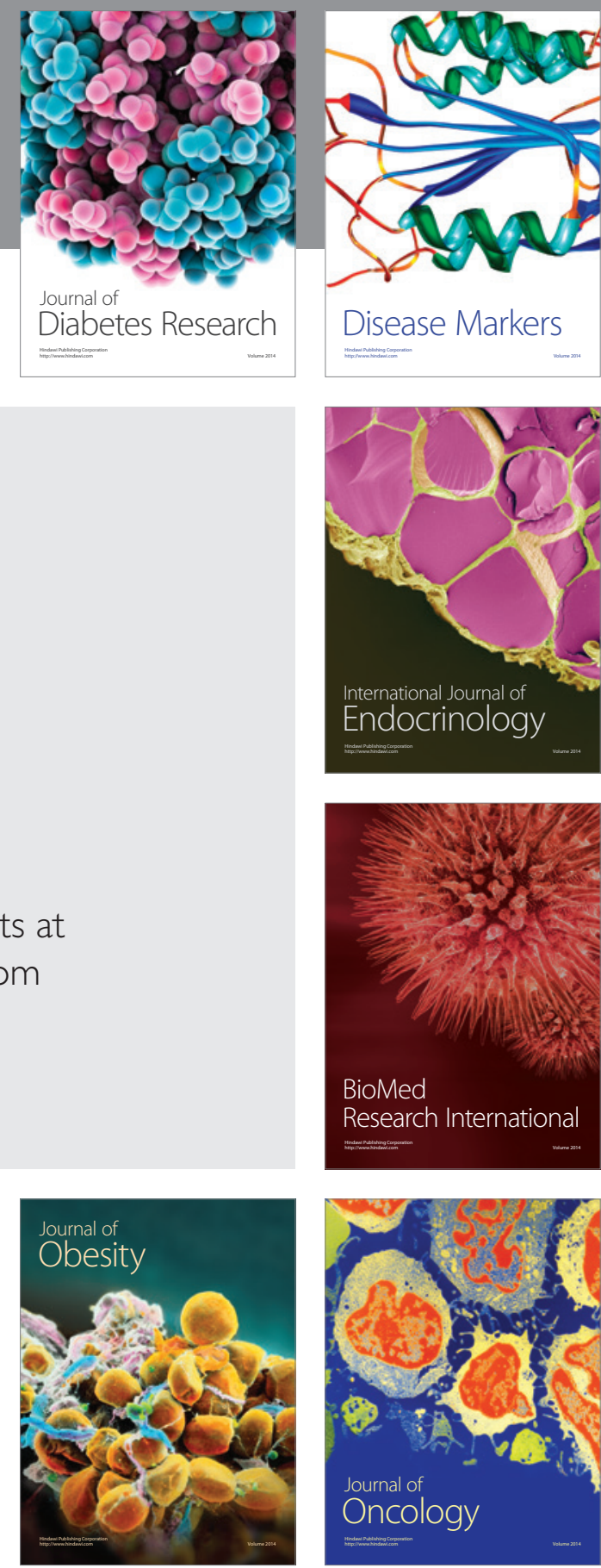

Disease Markers
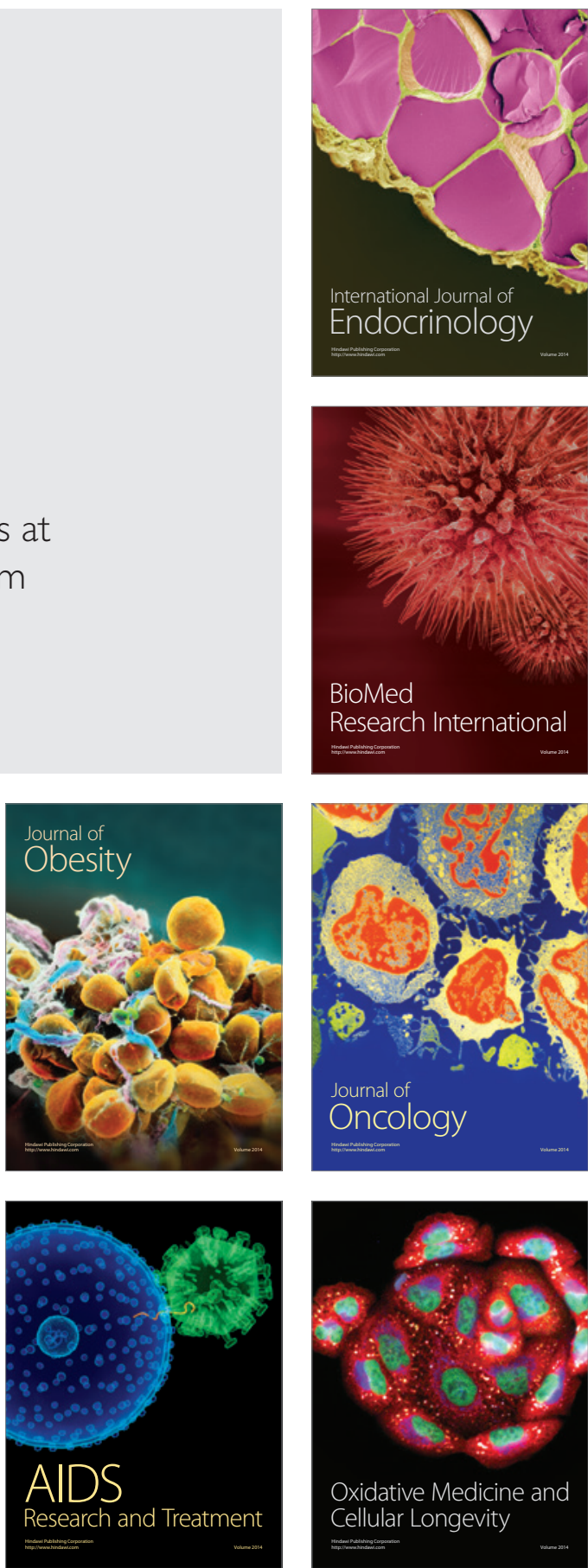